\title{
Filet mignon et steak haché ou le partage des coûts en forêt publique
}

\author{
by Yves Barrette, ing.f., M.Sc. et Marco Spain, c.a., c.m.a.
}

La nouvelle Loi sur les forêts, votée au Québec en 1986, a obligé les divers industriels forestiers s'approvisionnant dans un même territoire à intégrer leurs activités et à s'entendre sur le partage des coûts. La répartition du coût des infrastructures pose alors problème.

La pratique d'autrefois voulait que le premier industriel à exploiter le territoire construise et défraie la majeure partie, sinon la totalité du coût des chemins forestiers. Pour corriger cette inéquité, on est actuellement tenté d'utiliser la méthode du coût moyen imputé à chaque industriel; cette approche n'est pas plus équitable que la précédente et menace même la rentabilité de certains industriels.

Le présent article propose donc une troisième approche plus équitable: déjà employée dans d'autres secteurs d'activité, cette méthode basée sur la valeur de réalisation des produits est aussi appelée "'méthode du boeuf". Appliquée avec discernement, cette approche est de nature à minimiser les litiges actuels dans le partage des coûts rendu obligatoire par la nouvelle législation québécoise.
The new Quebec public Forests law, voted in 1986, has obliged forest enterprises using the same supplying territory to integrate their activities and to agree on cost sharing. Cost sharing of forest infrastructures can cause problems and disputes.

Until 1986, it was common practice for the first enterprise harvesting wood in a territory to build roads and bridges and pay for them partially or totally. Trying to solve the problem mentioned above and to correct that unfair practice, many are presently tempted to charge the average cost to each enterprise; this approach is no fairer than the preceding one and even threatens the profitability of some enterprises.

This article proposes a third approach that should be more appropriate; it is already used in at least one other activity sector and it is based on the products' realization value; it is familiarly called the "beef method". When applied with judgment, this approach can be useful to minimize the number of cases of cost sharing under dispute that are now mandatory under the new Quebec law.

\section{Introduction}

La nouvelle Loi sur les forêts, votée au Québec en 1986, a amené plusieurs industriels forestiers, s'approvisionnant dans un même territoire, à intégrer leurs activités, c'est-àdire à confier à l'un d'entre eux la récolte de tous les bois. Cette pratique est fort différente de celle qui prévalait généralement auparavant, alors que la récolte se faisait plutôt de façon successive par les divers industriels. Ainsi, en forêt mélangée, les bois résineux étaient d'abord récoltés, alors que la coupe des bois feuillus se faisait dans un second temps. Les industriels de bois résineux construisaient les infrastructures routières; les industriels de bois feuillus les utilisaient à peu ou pas de frais.

Aujourd'hui, les utilisateurs de résineux désirent que les utilisateurs de feuillus paient leur quote-part des infrastructures. Ils ne veulent plus, en tant que premiers utilisateurs des ressources d'un territoire, construire et défrayer seuls ou payer plus que leur quote-part du coût des chemins forestiers. On est alors tenté de répartir les coûts au prorata des volumes récoltés par chacun. Or, cette approche, qui est tout à fait à l'opposé de la précédente et qui apparaît logique, peut aussi être problématique. C'est pourquoi il sera ici question d'une troisième méthode de répartition des coûts qui respecte plus la valeur des différents bois: on devine, en effet, qu'une bille de bouleau jaune apte au déroulage vaut plus cher qu'un billot d'épinette et qu'elle peut, par conséquent, supporter une part plus élevée des coûts de récolte. La répartition des coûts constitue un problème d'actualité en forêt québécoise.

Les auteurs sont à l'emploi de la Société d'État québécoise Rexfor et sont notamment impliqués dans la résolution de problèmes liés à l'exploitation d'entreprises forestières.
Dans d'autres secteurs d'activité, le problème s'est présenté il y a déjà fort longtemps. L'exemple classique est celui du boeuf vendu à la boucherie: le kilo de filet mignon se vend beaucoup plus cher que celui de steak haché; pourtant ils proviennent du même boeuf élevé et acheté à un prix unique. Le boucher vend son steak haché moins cher qu'il a payé le boeuf; pourtant, s'il ne le vendait pas, il se priverait d'une source importante de revenus. Ce non-sens apparent a été réglé depuis longtemps en attribuant aux différentes parties du boeuf une part des coûts d'élevage, d'achat et de transformation de l'animal proportionnelle à leurs valeurs respectives, de façon à enregistrer une marge de bénéfice sur la vente de tous les produits découlant du boeuf. Cette méthode de répartition des coûts, fondée sur ce qu'il est convenu d'appeler la valeur de réalisation des produits, a pris en comptabilité le nom commun de "méthode du boeuf".

Pour bien comprendre comment s'appliquerait cette méthode en forêt et quelles sont ses conséquences, comparons-la avec les deux autres méthodes précitées à partir d'un exemple hypothétique simplifié.

\section{La méthode d'autrefois}

Trois industriels s'approvisionnant dans le même territoire requièrent respectivement les volumes suivants: $100000 \mathrm{~m}^{3}$ de résineux, $50000 \mathrm{~m}^{3}$ de sciage feuillu et $5000 \mathrm{~m}^{3}$ de déroulage feuillu. La méthode généralement utilisée avant l'avènement de la Loi sur les forêts au Québec, qui est celle des coûts marginaux successifs, chaque industriel faisant sa propre récolte de façon successive, aboutit à des résultats semblables à ceux présentés au tableau 1.

Les $155000 \mathrm{~m}^{3}$ récoltés dans notre exemple ont coûté en moyenne $44.22 \$ / \mathrm{m}^{3}$, dont $8.16 \$$ pour les infrastructures. Cependant, ces bois avaient une valeur moyenne au marché de $48.39 \$ / \mathrm{m}^{3}$, ce qui a laissé un bénéfice moyen de $8.60 \%$ sur l'opération. 
Tableau 1.

\begin{tabular}{lcccc}
\hline En $\$ \mathbf{~} \mathbf{m}^{\mathbf{3}}$ & $\begin{array}{c}\text { Bois } \\
\text { résineux }\end{array}$ & $\begin{array}{c}\text { Sciage } \\
\text { feuillu }\end{array}$ & $\begin{array}{c}\text { Déroulage } \\
\text { feuillu }\end{array}$ & Moyenne \\
\hline Coût des infrastructures & 11.00 & 3.00 & 3.00 & 8.16 \\
Autres coûts de récolte & 30.00 & 47.00 & 48.00 & 36.06 \\
Coût total de récolte & 41.00 & 50.00 & 51.00 & 44.22 \\
Valeur commerciale & 44.00 & 52.00 & 100.00 & 48.39 \\
Marge de bénéfice & 3.00 & 2.00 & 49.00 & 4.17 \\
& $6.82 \%$ & $3.85 \%$ & $49.00 \%$ & $8.60 \%$ \\
\hline
\end{tabular}

L'utilisateur de résineux qui, en construisant les chemins, a défrayé la majorité des coûts reliés aux infrastructures, a eu une marge de bénéfice de $6.82 \%$. Le scieur de bois feuillu n'a pas eu de frais élevés pour les infrastructures, mais le coût élevé de l'abattage et du débardage de ses bois ne lui a laissé que $3.85 \%$ de bénéfice. Le grand gagnant a été le dérouleur qui, à cause de la grande valeur au marché des billes de déroulage, a enregistré un bénéfice de $49 \%$.

Bien que les coûts de construction des infrastructures constituaient une bonne partie des coûts conjoints pour tous les industriels, aucune répartition entre les industriels n'était effectuée. Puisque la récolte s'effectuait de façon successive, chacun absorbait ses coûts propres; cela était simple, car tous les coûts étaient identifiables et contrôlables par l'industriel. Il est toutefois facile de concevoir que le premier industriel à développer les infrastructures forestières d'un secteur se voyait forcé de supporter une part souvent exagérée de ces coûts.

La nouvelle Loi sur les forêts du Québec amenant l'intégration des activités force les industriels à concevoir les coûts des infrastructures comme des coûts conjoints devant être répartis entre les différents utilisateurs. Voyons comment ce changement peut être traité.

\section{La méthode tentante}

Les résultats de la méthode d'autrefois engendrant une certaine inéquité, on est actuellement tenté d'utiliser une méthode de répartition de coûts basée sur les volumes récoltés par chacun, soit la méthode du coût moyen. Ainsi, le coût moyen de $8.16 \$ / \mathrm{m}^{3}$ pour les infrastructures, calculé au tableau précédent, est alors facturé à chaque industriel, chacun supportant alors une part égale. Voyons les implications de cette approche:

\section{Tableau 2.}

\begin{tabular}{lcccc}
\hline En $\$ / \mathbf{m}^{\mathbf{3}}$ & $\begin{array}{c}\text { Bois } \\
\text { résineux }\end{array}$ & $\begin{array}{c}\text { Sciage } \\
\text { feuillu }\end{array}$ & $\begin{array}{c}\text { Déroulage } \\
\text { feuillu }\end{array}$ & Moyenne \\
\hline Coût des infrastructures & 8.16 & 8.16 & 8.16 & 8.16 \\
Autres coûts de récolte & 30.00 & 47.00 & 48.00 & 36.06 \\
Coût total de récolte & 38.16 & 55.16 & 56.16 & 44.22 \\
Valeur commerciale & 44.00 & 52.00 & 100.00 & 48.39 \\
Marge de bénéfice & 5.84 & -3.16 & 43.84 & 4.17 \\
& $13.27 \%$ & $-6.08 \%$ & $43.84 \%$ & $8.60 \%$ \\
\hline
\end{tabular}

Cette approche, apparemment de gros bon sens, a amené un déficit d'opération au scieur de bois feuillu; en d'autres mots, il n'a tout simplement pas les moyens de payer une part équivalente à celle des autres industriels pour les infrastructures; à ce prix, il devrait se procurer ses bois ailleurs ou, à défaut, fermer boutique (on assume ici que la valeur commerciale est le prix maximal pour maintenir la rentabilité de l'entreprise). La méthode du coût moyen n'est donc pas plus équitable que celle des coûts marginaux successifs, puisqu'elle aurait pour effet à moyen terme d'occasionner la fermeture de certaines usines et ainsi augmenter les coûts de récolte des industriels restants, avec les conséquences que l'on peut imaginer.

Un partage égal des coûts comme celui ci-dessus peut donc finalement conduire à des situations non souhaitables pour tous. Il faut donc se pencher sur une autre méthode respectant chaque entité autant que l'ensemble. C'est sur cette prémisse que repose la troisième méthode présentée ci-après.

\section{La méthode du boeuf}

L'approche de la méthode du boeuf consiste à consentir la même marge de bénéfice à tous les produits, en l'occurrence $8.60 \%$ tel que calculé dans les tableaux précédents. Le coût des infrastructures imputable à chaque industriel est alors obtenu par différence:

Tableau 3.

\begin{tabular}{lcccc}
\hline En $\$ / \mathbf{m}^{3}$ & $\begin{array}{c}\text { Bois } \\
\text { résineux }\end{array}$ & $\begin{array}{c}\text { Sciage } \\
\text { feuillu }\end{array}$ & $\begin{array}{c}\text { Déroulage } \\
\text { feuillu }\end{array}$ & Moyenne \\
\hline Coût des infrastructures & 10.22 & 0.53 & 43.40 & 8.16 \\
Autres coûts de récolte & 30.00 & 47.00 & 48.00 & 36.06 \\
Coût total de récolte & 40.22 & 47.53 & 91.40 & 44.22 \\
Valeur commerciale & 44.00 & 52.00 & 100.00 & 48.39 \\
Marge de bénéfice & 3.78 & 4.47 & 8.60 & 4.17 \\
& $8.60 \%$ & $8.60 \%$ & $8.60 \%$ & $8.60 \%$ \\
\hline
\end{tabular}

Chaque industriel paie donc une part des infrastructures à la mesure de ses moyens: il s'agit sûrement là d'une approche plus équitable, le but final étant que tous les industriels demeurement en affaires.

\section{Commentaires et conclusion}

Les trois tableaux présentés ci-devant ont été simplifiés de façon à présenter une démarche. Cet article n'a pas la prétention de régler le débat actuel sur la répartition des coûts entre industriels forestiers; il vise plutôt à fournir une base de réflexion fondée sur une pratique ayant cours dans d'autres secteurs d'activité.

Plusieurs forestiers ont compris et appliqué depuis longtemps l'approche suggérée. Certains autres ne la connaissent pas ou préferrent attendre avant d'en tenir compte. Ceci est source de litiges et n'est évidemment pas de nature à simplifier la problématique de l'intégration des opérations forestières en forêt mélangée.

L'approche suggérée peut présenter certaines difficultés d'application; c'est pourquoi les industriels doivent s'en inspirer pour partager les coûts conjoints et non pas seulement l'appliquer intégralement. Les efforts de réflexion sur le sujet ne sont pas pour autant terminés, ni évidemment les négociations entre industriels. Les utilisateurs d'une forêt commune ont des intérêts communs et l'intégration de leurs activités de récolte suggère qu'ils doivent se voir comme des associés à qui la forêt doit également profiter.

Pour y parvenir, il ne faut pas oublier qu'on ne connaît pas de boucheries qui vendent le filet mignon au prix du steak haché. Et personne n'est prêt à acheter du steak haché d'un boucher qui le vend au prix du filet mignon. 